\title{
Pro-inflammatory markers in patients with obstructive sleep apnea and the effect of Continuous Positive Airway Pressure therapy
}

Jamil Al-Mughales ${ }^{1,2}$

Siraj Omar Wali ${ }^{3 *}$

Md. Dilshad Manzar ${ }^{4}$

Faris Alhejaili ${ }^{3}$

David Gozal ${ }^{5}$

\author{
${ }^{1}$ King Abdulaziz University Hospital, \\ Diagnostic Immunology Division, \\ Department of Clinical Laboratory \\ Medicine - Jeddah - Jeddah - Saudi Arabia. \\ ${ }^{2}$ Faculty of Medicine, King Abdulaziz \\ University, Department of Medical \\ Microbiology and Parasitology Immunology \\ Division - Jeddah - Jeddah - Saudi Arabia. \\ ${ }^{3}$ King Abdulaziz University, Sleep \\ Medicine and Research Center, Sleep \\ Medicine Research Group, Internal \\ Medicine Department, Faculty of \\ Medicine - Jeddah - Jeddah - Saudi \\ Arabia. \\ ${ }^{4}$ Majmaah University, Department of \\ Nursing, College of Applied Medical \\ Sciences - Majmaah - Majmaah - \\ Saudi Arabia. \\ ${ }^{5}$ The University of Missouri School of \\ Medicine, Department of Child Health \\ and the Child Health Research Institute - \\ Columbia - Columbia - United States.
}

*Corresponding author: Siraj Omar Wali

E-mail: sowali@kau.edu.sa

Received: November 12, 2020;

Accepted: March 1, 2021.

DOI: 10.5935/1984-0063.20200117

\begin{abstract}
Objectives: To evaluate the association of obstructive sleep apnea (OSA) with high-sensitivity C-reactive protein (CRP) and fibrinogen levels and to assess the effect of short-term therapy using continuous positive airway pressure (CPAP). Material and Methods: A prospective, openlabel, controlled trial was conducted among clinically referred patients at risk for OSA undergoing diagnostic polysomnography (PSG). After PSG, the patients were divided into 3 groups: OSA treatment group (TG) $(n=21)$, untreated OSA group (UOG) $(n=19)$, and non-OSA healthy control group (HCG) ( $\mathrm{n}=24)$. CRP and fibrinogen levels were measured at baseline and one month after treatment. Repeated-measures (RM) ANOVA and ANCOVA were used to compare changes in CRP and fibrinogen levels among the three groups by analyzing between-subject and within-subject effects as functions of time and adjusting for significant covariates. Results: At baseline, OSA subjects had significantly higher CRP $[\mathrm{t}(52.37)=-2.46, \mathrm{p}=0.02)]$ and fibrinogen levels $[\mathrm{t}(57)=-2.00$, $\mathrm{p}=0.05)]$ than HCG subjects. No significant differences in CRP levels $[(\mathrm{F}(2,58)=2.29, \mathrm{p}=0.11)]$ or fibrinogen levels $[(\mathrm{F}(2,58)=1.28, \mathrm{p}=0.29)]$ emerged between TG and HCG subjects after adjusting for the pretest levels. Conclusion: CPAP therapy for one month does not affect CRP and fibrinogen levels among moderate-to-severe OSA patients. However, OSA is associated with elevated levels of these inflammatory biomarkers.
\end{abstract}

Keywords: Inflammation; Sleep Apnea Syndromes; C-Reactive Protein; Fibrinogen. 


\section{INTRODUCTION}

Obstructive sleep apnea (OSA) is a common sleeprelated breathing disorder that is characterized by repeated complete or partial collapse of the upper airway during sleep $^{1-3}$. OSA results in momentary intermittent hypoxemia and hypercapnia, sleep fragmentation, and poor sleep quality ${ }^{4}$. OSA has clear negative impacts on the quality of life of affected individuals. This condition is also associated with adverse safety and health consequences, including cardiovascular and cerebrovascular diseases, type 2 diabetes, cognitive impairments, depression, and ocular conditions ${ }^{5,6}$. Moreover, OSA is a risk factor for cardiovascular disease, probably due to increases in systemic inflammation and oxidative stress and their injurious effects on the vascular endothelium ${ }^{7}$. This status of increased systemic inflammation may result in initiation and acceleration of underlying atherosclerosis with consequent increases in morbidity and mortality ${ }^{8,9}$. Indeed, the recent literature supports a conceptual framework wherein OSA should be considered a low-grade chronic systemic inflammatory disorder ${ }^{10}$.

Previous studies proposed that OSA modulates the expression and secretion of inflammatory markers from fat and other tissues ${ }^{10,11}$. In fact, independent of obesity, elevated levels of proinflammatory factors, including C-reactive protein (CRP), fibrinogen, tumor necrosis factor- $\alpha$ and interleukin- 6 , have been reported among patients with $\mathrm{OSA}^{10}$. Hence, ongoing inflammatory responses have been suggested to play important roles in the association between OSA and chronic inflammationinduced pathologies, such as atherosclerosis ${ }^{12}$. The activation of inflammatory responses through adaptive pathways in OSA may be an important molecular mechanism underlying the development of cardiovascular disease and a variety of other metabolic diseases ${ }^{13,14}$.

Continuous positive airway pressure (CPAP) remains the most effective therapy to date for improving the polysomnographyderived parameters indicating OSA severity, i.e., the apnea hypopnea index (AHI), oxygen desaturation index 3\% (ODI3) and nadir oxyhemoglobin saturation $\left(\mathrm{SaO}_{2}\right)^{4,15,16}$. Thus, exploring the potential relationships between systemic inflammatory markers in OSA and the effect of CPAP-based treatment is important. There are conflicting data in the literature regarding the efficacy of CPAP in reducing the levels of proinflammatory biomarkers and, consequently, providing an overall protective effect against the development of atherosclerosis and cardiovascular diseases ${ }^{17-21}$. This study was conducted to explore the effect of short-term CPAP therapy on the levels of two well-established proinflammatory biomarkers, namely, CRP and fibrinogen, which are regarded as biological indicators of ongoing systemic inflammation.

\section{MATERIAL AND METHODS}

\section{Design and setting}

This was a prospective controlled trial that was conducted from April 2018 to May 2019. The trial took place at the Sleep Medicine and Research Center (SMRC) at King Abdul-Aziz University Hospital (KAUH), Jeddah, Saudi Arabia.

\section{Population}

All patients with a clinical suspicion of OSA who were referred to the SMRC for diagnostic polysomnography (PSG) were included in the study. The exclusion criteria included a past diagnosis of OSA, treatment for OSA, presence of conditions that could affect the levels of inflammatory markers, including history of respiratory and cardiovascular diseases with chronic hypoxia, neuromuscular disorders, infectious diseases, rheumatological diseases, immunological diseases, tumors, peripheral vascular disease, liver or kidney diseases, coagulopathy, psychogenic disorders, and diabetes mellitus; history of trauma or surgery in the past 3 months; recent use (past 3 months) of corticosteroids, antibiotics, immune suppressors, or hormones; and smoking history in the past 6 months.

\section{Procedures}

Initial assessment

All patients referred to the SMRC for a sleep study underwent data collection and physical examination. Demographic data, including age, sex, body mass index (BMI), neck circumference, and blood pressure, were collected. The Epworth sleepiness scale (ESS) was used to assess daytime sleepiness ${ }^{22}$. All eligible subjects were invited to participate and signed an informed consent form that was approved by the local institutional review board (IRB) (reference number 178-18).

\section{Overnight PSG}

All participants underwent overnight PSG using a standard montage, as previously described ${ }^{23}$. The sleep stages and respiratory events were scored according to the guidelines of the American Academy of Sleep Medicine (AASM) ${ }^{24}$. The severity of OSA was determined according to the AASM recommendations using the AHI: 5-14, mild; 15-29, moderate; and $\geq 30$, severe $^{25}$.

\section{Group allocation}

According to the PSG results, participants were categorized into 3 groups:

The first group included those with moderate-tosevere OSA according to the AASM definition ${ }^{25}$, i.e., those with an AHI of 15/hour of total sleep time (hr'TST) or greater, who accepted initiation treatment with CPAP (treatment group [TG]). The second group included patients with moderateto-severe OSA who refused CPAP treatment (untreated OSA group [UOG]). The third group included participants without OSA, i.e., those with an AHI less than 5 (healthy control group [HCG]) (Figure 1).

\section{Initial proinflammatory marker measurements}

Fasting blood samples were collected from all study participants to measure the serum and plasma levels of proinflammatory markers in the morning after the diagnostic PSG. Venous blood samples were collected into $5 \mathrm{ml}$ tubes with 


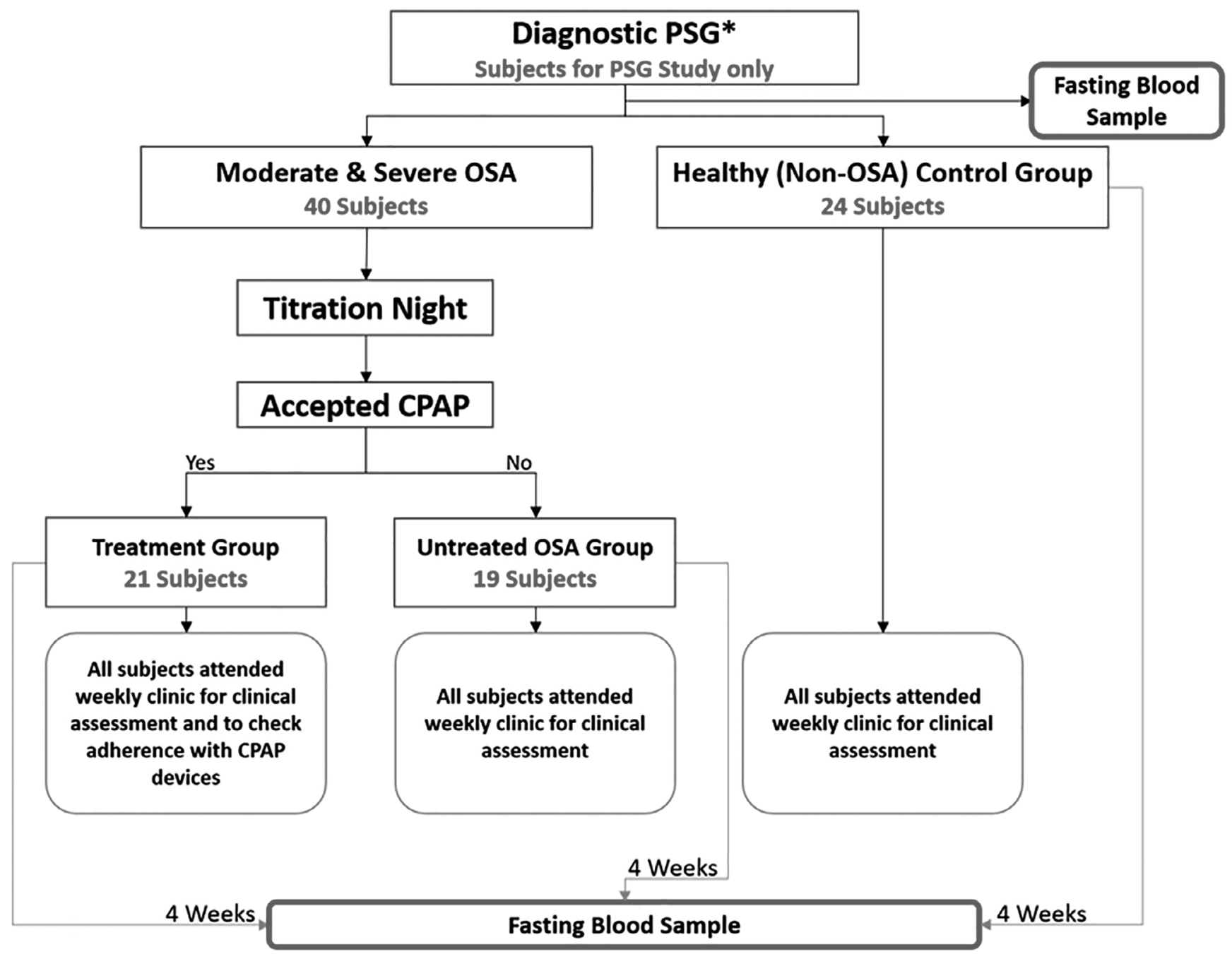

Figure 1. Flowchart of the study procedure.

and without added anticoagulation factors, and the serum and plasma were separated and stored at $-80^{\circ} \mathrm{C}$ immediately.

Commercially available assays were used to quantify highsensitivity CRP (Siemens; Germany) and fibrinogen (ACL TOP 550 CTS Instrumentation Laboratory, Italy) levels according to the manufacturer's instructions. The CRP and fibrinogen assays exhibited analytical sensitivities less than $3.4 \mathrm{mg} / 1$ and $50 \mathrm{mg} / \mathrm{dl}$, respectively. Serum CRP concentrations were measured using fully automated BNII nephelometer (Siemens; Germany). The cutoff value for a normal CRP level was $3.4 \mathrm{mg} / \mathrm{l}$. Fibrinogen was measured in citrated plasma by an ACL TOP 550 instrument (Instrumentation Laboratory, Italy). The measurement range was between 180 and 350mg/dl. Plasma heparin concentrations below $2 \mathrm{U} / \mathrm{ml}$ did not affect the test. Concentrations of CRP $>3.4 \mathrm{mg} / 1$ and fibrinogen $>350 \mathrm{mg} / \mathrm{dl}$ were considered elevated.

\section{CPAP THERAPY}

All participants with OSA who agreed to initiate treatment underwent a CPAP titration PSG study to determine the optimal CPAP pressure according to the AASM guidelines ${ }^{19}$, and this pressure was then implemented at home.

\section{Follow-up and endpoints}

The three groups were followed for one month following the diagnostic PSG. Follow-up included weekly visits to determine whether there were any necessary changes to the medications or events that could affect the levels of the inflammatory markers and to ensure adherence to CPAP treatment in the TG using data from memory cards installed within the CPAP devices ${ }^{26}$. Adherence to CPAP was considered acceptable when the minimum CPAP use was $\geq 4$ hours $/ 70 \%$ of the nights. At the end of the one-month follow-up, fasting morning blood samples were drawn to analyze the levels of the proinflammatory biomarkers, which were processed as described above. Participants who did not meet the adherence criteria were excluded.

\section{Statistical methods}

All of the data analyses in this study were performed using the Statistical Package for Social Sciences version 26.0 
for Windows (SPSS Inc., Chicago, IL, USA). Means, standard deviations, frequencies and percentages were used to describe the data. Chi-square tests, maximum likelihood ratio chisquare tests, and one-way and Kruskal-Wallis tests were used to show differences in the participants' characteristics. CRP and fibrinogen levels were not normally distributed and were therefore log-transformed for further analysis. One-way ANOVA and one-way ANCOVA were used to assess differences in the log-transformed CRP and log-transformed fibrinogen values. Principal component analysis was used to estimate a single common component (age, BMI, neck circumference, AHI, and sex) to use as a covariate to adjust one-way ANCOVA. Binary logistic regression was used to assess association between OSA, inflammatory markers, and other factors. The model was explored with OSA condition (yes/no) as dependent variable and variables such as sex, age, BMI, neck circumference, systolic blood pressure (BP), diastolic BP, ESS score, STOP-BANG classification, log-transformed CRP, and log-transformed fibrinogen as independent variables. A two-tailed $p$-value $<0.05$ was considered statistically significant.

\section{RESULTS}

\section{Participant characteristics}

The baseline characteristics of the study participants are shown in Table 1. There was no significant difference in sex distribution among the three groups $(\mathrm{p}=0.26)$; however, $\mathrm{TG}$ subjects were significantly older (51.9 \pm 12.9 years) than UOG (43.1 \pm 12.5 years) and HCG $(29.6 \pm 8.8$ years $)$ subjects $(\mathrm{p}<0.01)$. Additionally, BMI $(\mathrm{p}=0.04)$ and neck circumference $(\mathrm{p}<0.01)$ differed significantly among the three groups, whereas ESS score $(\mathrm{p}=0.52)$ and $\mathrm{BP}$ (systolic: $\mathrm{p}=0.27$; diastolic: $\mathrm{p}=0.12$ ) were similar among groups. There were no statistically significant differences among the three groups in log-transformed CRP values $(\mathrm{F}(2,58)=2.45, \mathrm{p}=0.10)$ or log-transformed fibrinogen values $(\mathrm{F}(2,58)=1.99, \mathrm{p}=0.14)$.

Table 1. Characteristics of the subjects in the three groups.

\begin{tabular}{l|cccc}
\hline Characteristics & HCG & TG & UOG & $\begin{array}{c}\text { P } \\
\text { value }\end{array}$ \\
\hline Gender & & & & \\
Female & $12(60.0 \%)$ & $7(35.0 \%)$ & $8(42.1 \%)$ & .263 \\
Male & $8(40.0 \%)$ & $13(65.0 \%)$ & $11(57.9 \%)$ & \\
Age & $29.6 \pm 8.77$ & $51.95 \pm 12.89$ & $43.10 \pm 12.51$ & $<.01$ \\
BMI & $30.40 \pm 9.43$ & $36.16 \pm 12.08$ & $39.35 \pm 11.02$ & .04 \\
Neck & $34.18 \pm 5.91$ & $38.24 \pm 4.51$ & $40.16 \pm 3.26$ & .001 \\
circumference & & & & \\
Blood pressure & $131.7 \pm 16.62$ & $134.00 \pm 14.26$ & $142.24 \pm 28.32$ & .270 \\
Systolic & $72.45 \pm 7.82$ & $78.68 \pm 18.33$ & $86.24 \pm 29.46$ & .122 \\
Diastolic & & & & \\
STOP-BANG & $13(65.0 \%)$ & $4(20.0 \%)$ & $5(26.3 \%)$ &. \\
Low risk & $2(10.0 \%)$ & $7(35.0 \%)$ & $3(15.8 \%)$ & 018 \\
Intermediate risk & $5(25.0 \%)$ & $9(45.0 \%)$ & $11(57.9 \%)$ & \\
High risk & $13.60 \pm 6.25$ & $11.40 \pm 6.18$ & $12.16 \pm 6.06$ & .523 \\
ESS & $2.52 \pm 1.85$ & $40.78 \pm 26.80$ & $32.98 \pm 20.25$ & $<.01$ \\
\hline AHI & & & & \\
\hline
\end{tabular}

Baseline differences in fibrinogen and CRP levels among OSA participants

At baseline, participants with OSA had significantly higher baseline serum log-transformed CRP levels $(0.80 \pm 0.35 \mathrm{mg} / \mathrm{dl})$ than HCG subjects $(0.61 \pm 0.24 \mathrm{mg} / \mathrm{dl}$; actual CRP values: $6.35 \pm 2.24 \mathrm{mg} /$ dl versus $4.10 \pm 1.73 \mathrm{mg} / \mathrm{dl}, \mathrm{t}(52.37)=-2.46, \quad p=0.02)$. Similarly, participants with OSA had significantly higher baseline serum logtransformed fibrinogen levels $(2.51 \pm 0.12 \mathrm{mg} / \mathrm{dl})$ than HCG subjects $(2.45 \pm 0.12 \mathrm{mg} / \mathrm{dl}$; actual fibrinogen levels: $323.59 \pm 1.32 \mathrm{mg} / \mathrm{dl}$ versus $281.84 \pm 1.32 \mathrm{mg} / \mathrm{dl}, \mathrm{t}(57)=-2.00, p=0.05)$.

Baseline differences between the OSA groups: age, BMI, AHI, neck circumference, and STOP-BANG classification

Age was significantly higher in the TG $(51.95 \pm 12.89$ years $)$ than in the UOG $(43.10 \pm 12.51$ years, $\mathrm{t}(37)=2.17, p=0.04$ ) (Table 2 ).

Table 2. Baseline differences in age and AHI between OSA groups.

\begin{tabular}{lccc}
\hline \multirow{2}{*}{ Characteristics } & \multicolumn{2}{c}{ Mean \pm SD/number (percentage) } & \\
& TG $(\mathbf{n}=\mathbf{2 1})$ & UOG $(\mathbf{n = 1 9 )}$ & p-value \\
\hline Age (years) & $51.95 \pm 12.89$ & $43.10 \pm 12.51$ & 0.036 \\
AHI & $40.78 \pm 26.80$ & $32.98 \pm 20.25$ & 0.31 \\
BMI & $36.16 \pm 12.08$ & $39.35 \pm 11.02$ & 0.395 \\
Neck & $38.24 \pm 4.51$ & $40.16 \pm 3.27$ & 0.139 \\
circumference & & & \\
STOP-BANG & & $5(26.3 \%)$ & \\
Low risk & $4(20.0 \%)$ & $3(15.8 \%)$ & 0.381 \\
Intermediate risk & $7(35.0 \%)$ & $11(57.9 \%)$ & \\
High risk & $9(45.0 \%)$ & & \\
\hline
\end{tabular}

\section{Baseline differences in fibrinogen and CRP levels between the treated and untreated OSA groups}

There were no significant differences in the $\log$ transformed CRP levels $(\mathrm{F}(1,39)=0.17, p=0.68)$ and $\log$ transformed fibrinogen levels $(F(1,39)=2.45, p=0.86)$ between the TG and UOG groups at baseline, even after adjusting for covariates, including age, BMI, neck circumference, and AHI (log-transformed CRP: $(\mathrm{F}(1,39)=0.16, p=0.69$; log-transformed fibrinogen: $\mathrm{F}(1,39)=0.02, p=0.88)$ (Table 3).

Table 3. Fibrinogen and CRP levels in the treated and nontreated OSA groups.

\begin{tabular}{l|cccc}
\hline $\begin{array}{l}\text { Serum inflammatory } \\
\text { marker level }\end{array}$ & $\begin{array}{c}\text { MG } \\
(\mathrm{n}=21)\end{array}$ & $\begin{array}{c}\text { UOG } \\
(\mathrm{n}=19)\end{array}$ & $\begin{array}{c}\text { p-value } \\
\text { (unadjusted) }\end{array}$ & $\begin{array}{c}\text { p-value } \\
\text { (adjusted) }\end{array}$ \\
\hline $\begin{array}{l}\text { Log-transformed CRP } \\
(\mathrm{m} / \mathrm{dl})\end{array}$ & $\begin{array}{c}0.78 \pm \\
0.37\end{array}$ & $\begin{array}{c}0.83 \pm \\
0.34\end{array}$ & 0.679 & 0.689 \\
Log-transformed & & & & \\
fibrinogen $(\mathrm{mg} / \mathrm{dl})$ & $\begin{array}{c}2.51 \pm \\
0.13\end{array}$ & $\begin{array}{c}2.52 \pm \\
0.12\end{array}$ & 0.863 & 0.875 \\
\hline
\end{tabular}

A common component score that could be used as a covariate in the model was generated using principal component analysis for age, BMI, neck circumference, AHI, and sex. This common component score explained $34.21 \%$ of the variability in age, BMI, neck circumference, $\mathrm{AHI}$ and sex. The homogeneity 
of the regression condition was determined to be satisfactory, with a non-significant interaction between groups $(\mathrm{F}(1,39)=2.61 ; p=0.12)$ and log-transformed CRP as the dependent variable. Similarly, there was no significant interaction between groups $(\mathrm{F}(1,39)=1.76$; $p=0.19)$, with log-transformed fibrinogen as the dependent variable.

\section{Association of inflammatory markers and other variables with OSA at baseline}

The binary logistic regression model for OSA was statistically significant when compared to the model with only intercepts; $\chi^{2}(11, \mathrm{~N}=59)=43.44, p<0.01$. The prediction model with 10 factors accounted for $74.1 \%$ (Nagelkerke R Square) of the variance in the classification of OSA. The model correctly classified $89.3 \%$ of the cases (sensitivity $=94.4 \%$ and specificity $=80.0 \%$ ), with increasing age being associated with an increasing likelihood of OSA (adjusted odds ratio $(\mathrm{AOR})=1.26$, 95\% confidence interval (CI): 1.07-1.48; $p<0.01)$.

\section{CRP levels: within-group pre-post difference}

At the 1-month follow-up, the log-transformed CRP levels were significantly higher in the HCG group than in the baseline group $(0.68 \pm 0.26 \mathrm{mg} / \mathrm{dl}$ vs. $0.61 \pm 0.24 \mathrm{mg} / \mathrm{dl} ; \mathrm{t}(19)=-3.07, p=0.01)$. In addition, CPAP treatment was not associated with any statistically significant differences in CRP levels (Figure 2).

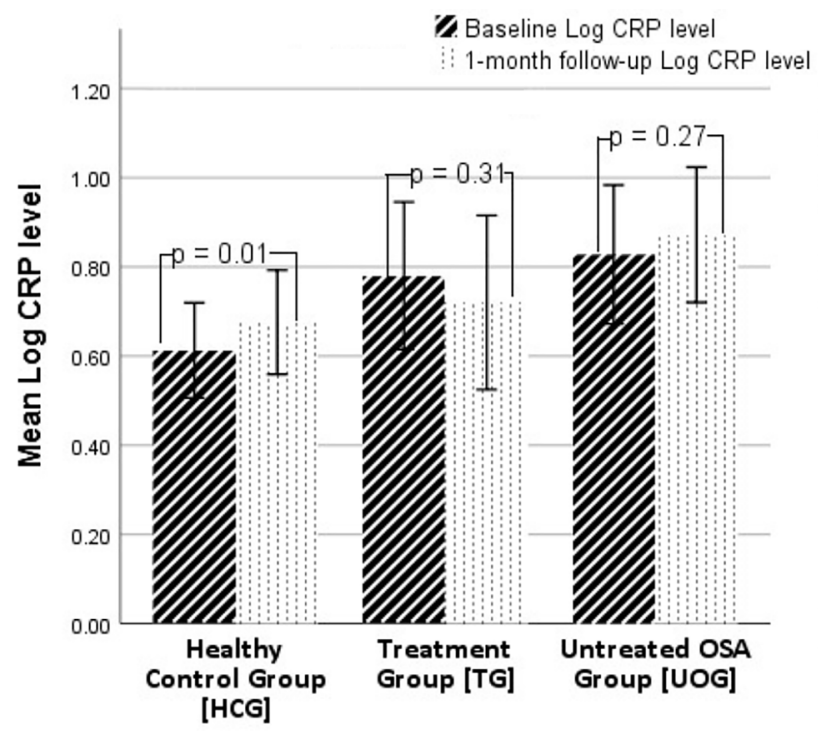

Figure 2. Mean CRP levels at baseline and at 1-month follow-up in the HCG and the two OSA groups. TG: OSA Treatment Group, UOG: Untreated OSA Group, HCG: Healthy Control Group.

\section{Fibrinogen levels: within-group pre-post difference}

At the 1-month follow-up, the fibrinogen levels were significantly increased in the HCG compared with that at baseline $(2.48 \pm 0.10 \mathrm{mg} / \mathrm{dl}$ vs. $2.45 \pm 0.12 \mathrm{mg} / \mathrm{dl}$ at baseline; $\mathrm{t}(19)=-2.13$, $p=0.05$ ) (Figure 2). No significant changes after CPAP treatment emerged in fibrinogen level in the TG subjects $(p=0.87)$, and similar to the HCG, the UOG also exhibited significant increases in fibrinogen levels at the 1 -month follow-up $(2.55 \pm 0.10 \mathrm{mg} / \mathrm{dl}$ vs. baseline: $2.52 \pm 0.12 \mathrm{mg} / \mathrm{dl} ; \mathrm{t}(18)=-2.17, p=0.04$ ) (Figure 3).

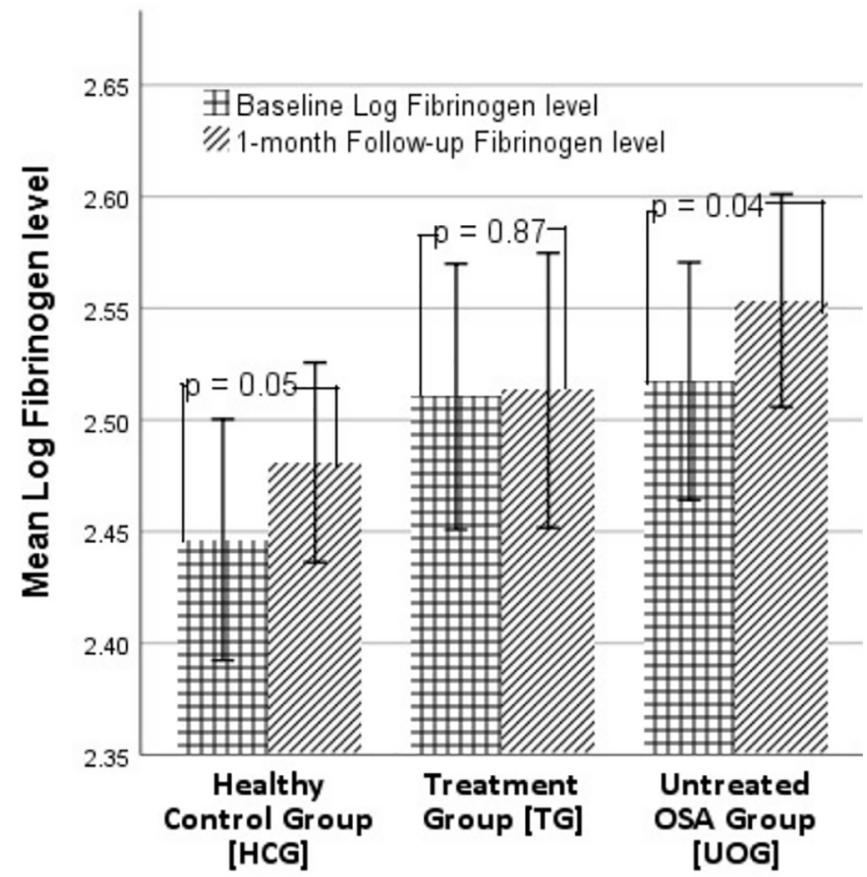

Figure 3. Mean fibrinogen levels at baseline and at 1-month follow-up in the HCG, TG and UOG groups. TG: OSA Treatment Group, UOG: Untreated OSA Group, HCG: Healthy Control Group.

\section{Effect of 1 month of CPAP therapy on CRP and fibrinogen levels}

ANCOVA revealed that there were no significant differences in the log-transformed fibrinogen levels between the treated group and the untreated groups after 1 month of CPAP therapy after adjusting for baseline levels $(\mathrm{F}(2,58)=1.28$, $p=0.29)$, and similar findings emerged for the log-transformed CRP levels $(\mathrm{F}(2,58)=2.29, p=0.11)$.

\section{DISCUSSION}

Increased levels of CRP and fibrinogen are detectable among OSA patients, supporting the conceptual framework that OSA is a chronic low-grade systemic inflammatory condition. However, adherence to CPAP treatment for one month did not lead to significant changes in these inflammatory markers. Nevertheless, while a significant increase in the fibrinogen level after 4 weeks of follow-up was observed in both the HCG and untreated OSA group compared to baseline, no such increases were detectable in the CPAP-treated subjects, suggesting that rather than reversing the expression of inflammatory biomarkers, CPAP treatment would be expected to abrogate the temporal progression of inflammation.

The current study supports previous published findings indicating that OSA is a chronic inflammatory condition ${ }^{10,14}$. Nadeem et al. $(2013)^{10}$ conducted a meta-analysis that included 51 studies and concluded that the levels of inflammatory markers are higher in patients with OSA than in control subjects. Furthermore, Li et al. $(2017)^{14}$, in another metaanalysis, reported the link between serum CRP levels and OSA and the interactive effects of obesity and the severity of OSA on CRP concentrations. More recently, however, a Korean study involving 1,835 subjects showed that CRP levels were elevated 
in patients with moderate and severe OSA, independent of other confounders, including obesity ${ }^{27}$. It is worth mentioning that although the severity of OSA is usually classified by the AHI according to $\mathrm{AASM}^{25}$, however, other parameters may also determine OSA severity, such as oxygen saturation ${ }^{28}$. Accordingly, CRP levels was reported to correlate significantly not only with AHI but also with oxygen saturation during sleep $^{29}$. In addition, Kim et al. (2016) ${ }^{27}$ reported that CRP levels were negatively associated with the $\mathrm{SaO}_{2}$ nadir. Nevertheless, despite the presence of elevated levels of inflammatory markers, including CRP and fibrinogen, among OSA patients, the association between the levels of inflammatory markers and OSA severity has proven elusive and inconsistent ${ }^{30-33}$. The tenacious investigation of these biomarkers in the context of OSA is obviously explained by the fact that these markers are strongly associated with cardiovascular risk and endothelial dysfunction, as well as with metabolic syndrome and stroke ${ }^{34-36}$.

In the present study, short-term treatment consisting of one month of adherence to CPAP therapy did not lead to obvious reductions in the levels of these inflammatory markers. Several inflammatory markers that are believed to contribute to the pathogenesis of endothelial dysfunction have been previously studied among OSA patients before and after CPAP therapy ${ }^{18,37}$. In line with our findings, a controlled trial tested the effect of CPAP therapy on fibrinogen levels and erythrocyte sedimentation rate (ESR) in patients diagnosed with OSA and cardiac arrhythmias and found no significant differences after 3 and 6 months of treatment compared to the corresponding values in patients who received only pharmacological treatment ${ }^{38}$. The authors concluded that fibrinogen and ESR may not be reliable markers of the efficacy of CPAP therapy ${ }^{38}$. Similarly, a cohort study measured the post-CPAP therapy changes in both fibrinogen and CRP levels in patients with OSA, including those with ischemic heart disease (IHD), and found no differences in the two markers after 3 months of CPAP therapy ${ }^{39}$. However, when analyzing the effect of CPAP treatment on OSA patients without the clinical manifestations of IHD, a statistical trend towards a decrease in mean CRP levels was observed $(\phi=0.05)$. These findings may suggest that a more beneficial effect of CPAP therapy should be expected among OSA patients without clinically apparent $\mathrm{IHD}^{39}$. We should point out that evidence for the reversibility of vascular inflammation after long-term exposure to intermittent hypoxia mimicking sleep apnea was not apparent in a mouse model and was likely explained by epigenetic changes in macrophage pathways underlying sustained inflammatory processes ${ }^{40,41}$.

Conversely, a meta-analysis that included nearly 1,200 OSA patients from 14 cohort studies found standardized mean differences of 0.68 and 0.74 units in CRP levels after 3 and 6 months of CPAP therapy, respectively, compared to the preCPAP therapy measurements ${ }^{19}$. The authors concluded that CRP was a reliable indicator of the efficacy of CPAP therapy and that the use of CRP concentrations could assist in the prediction of cardiovascular risk in OSA patients ${ }^{19}$. Another research team investigated the effect of 6 months of nasal
CPAP therapy on CRP levels in patients with overlap syndrome, i.e., the coexistence of OSA with chronic obstructive pulmonary disease (COPD). The findings showed a nearly 50\% decrease in the mean CRP levels from baseline, and the decline in CRP levels was linearly correlated with the number of hours of CPAP therapy utilized per night ${ }^{42}$. These findings concur with those of a similar trial that tested the effect of CPAP therapy on systemic inflammatory markers in patients with overlap syndrome and in those with OSA alone ${ }^{43}$. Nural et al. (2013) $)^{43}$ reported significant CPAP-induced decreases in CRP levels in both the overlap syndrome and OSA groups $(p=0.04$ and $p=0.02$, respectively). Sex-specific effects of CPAP therapy on CRP levels have also been postulated, whereby a better effect on CRP level may be present among males receiving 3 months of CPAP treatment, as compared to the unaltered CRP levels among females after 3 months of the same treatment. Of note, female patients required 6 months of CPAP therapy to manifest declines in CRP concentrations ${ }^{44}$. The discrepancy between the results of these studies and our current findings could be related to the shorter duration of CPAP in our study or could be ascribed to differences among the participant cohorts, since we excluded patients with factors that could have exacerbated their systemic inflammatory status. Nevertheless, these observations suggest that a favorable effect of CPAP may occur in terms of fibrinogen levels, as well as CRP concentrations, provided that the treatment is adhered to for a much longer period of time. More importantly, we surmise that the beneficial effects of CPAP therapy may be more apparent when illustrated by CRP levels in specific patient subgroups (i.e., men with relatively severe OSA and without clinically established cardiovascular conditions).

Similar to that of other studies, the purpose of the present study was to determine the therapeutic effect of CPAP therapy on systemic inflammation, with the ultimate goal of reducing the global cardiovascular risk among OSA patients. However, the only favorable finding supporting such assumption resides in the indirect effect of CPAP therapy, whereby treatment was accompanied by the absence of an increase in fibrinogen and CRP levels in the treated group. Notwithstanding, we cannot rule out that such changes may also be due to a cyclic or seasonal variation in inflammation biomarkers that is prevented by CPAP therapy, since notable seasonal variations were reported in a large population-based study of inflammatory biomarkers ${ }^{45}$.

There are several aspects and limitations of this study that should be highlighted. First, the one-month duration of CPAP therapy may not have been sufficient to induce a significant change in the plasma markers of inflammation. Second, the small sample size of the study groups may have hampered the detection of significant differences due to type 2 error. Third, due to the absence of randomization, notable selection bias may have occurred during group allocation, in which older patients and those with more severe OSA were more likely to be included in the CPAP treatment group. Fourth, patients with a CPAP use of $\geq 4$ hours $/ 70 \%$ of the nights was considered acceptable and included in the study. However, this adherence variable was not 
extracted further as level of adherence. Therefore, relationship between OSA and short-term CPAP could not be reported after adjusting for adherence level. Future studies may be better placed to record adherence level of CPAP, severity of OSA in groups, etc. All of these factors may have resulted in a reduction in the relative effect of CPAP therapy.

\section{CONCLUSION}

CPAP therapy for one month does not affect CRP and fibrinogen levels among moderate-to-severe OSA patients. However, one month of adherence to CPAP therapy may have a favorable impact on CRP and fibrinogen levels in moderateto-severe OSA patients by preventing temporal increases in such markers. Furthermore, our study confirms that OSA is associated with elevated levels of such inflammatory biomarkers.

\section{ACKNOWLEDGEMENTS}

We are grateful to Dr. Afnan Hafiz and Dr. Ibrahim Zakaria for their help in the data collection process. The authors would also like to thank Mr. Badrudin Banjar and Mrs. Ofelia El-Assal from the Immunology Lab, King Abdulaziz University Hospital for their help in collecting and analyzing the blood samples. Finally, we would like to acknowledge the great efforts made by Mrs. Walaa Abuzahra, Research Coordinator, Sleep Medicine and Research Center, in coordinating all study procedures.

\section{FUNDING}

This project was funded by the Deanship of Scientific Research (DSR), King Abdulaziz University, Jeddah, Saudi Arabia under grant No. (KEP-2-140-39). The authors, therefore, acknowledge and appreciate the DSR for their technical and financial support.

\section{REFERENCES}

1. Dempsey JA, Veasey SC, Morgan BJ, O'Donnell CP. Pathophysiology of sleep apnea. Physiol Rev. 2010 Jan;90(1):47-112.

2. Benjafield AV, Ayas NT, Eastwood PR, Heinzer R, Ip MSM, Morrell MJ, et al. Estimation of the global prevalence and burden of obstructive sleep apnoea: a literature-based analysis. Lancet Respir Med. 2019 Aug;7(8):687-98.

3. Wali SO, Abalkhail B, Krayem A. Prevalence and risk factors of obstructive sleep apnea syndrome in a Saudi Arabian population. Ann Thorac Med. 2017 Apr/Jun;12(2):88-94.

4. Lévy P, Kohler M, McNicholas WT, Barbé F, McEvoy RD, Somers VK, et al. Obstructive sleep apnoea syndrome. Nat Rev Dis Primers. 2015 Jun;1(1):15015.

5. Lee W, Nagubadi S, Kryger MH, Mokhlesi B. Epidemiology of obstructive sleep apnea: a population-based perspective. Expert Rev Respir Med. 2008 Jun;2(3):349-64.

6. Young T. Rationale, design and findings from the Wisconsin Sleep Cohort Study: toward understanding the total societal burden of sleep disordered breathing. Sleep Med Clin. 2009 Mar;4(1):37-46.

7. Kohli P, Balachandran JS, Malhotra A. Obstructive sleep apnea and the risk for cardiovascular disease. Curr Atheroscler Rep. 2011 Apr;13(2):138-46.

8. Colla-Machado PE, Luzzi AA, Balian NR, Pigretti SG, ZurrúGanen MC, Cristiano E, et al. Prevalence of silent brain infarction in obstructive sleep apnea. Nat Clin Pract Neurol. 2007;3(7):362.

9. Maeder MT, Schoch OD, Rickli H. A clinical approach to obstructive sleep apnea as a risk factor for cardiovascular disease. Vasc Health Risk Manag. 2016 Mar;12:85-103.

10. Nadeem R, Molnar J, Madbouly EM, Nida M, Aggarwal S, Sajid H, et al. Serum inflammatory markers in obstructive sleep apnea: a metaanalysis. J Clin Sleep Med. 2013 Oct;9(10):1003-12.

11. Sharma SK, Mishra HK, Sharma H, Goel A, Sreenivas V, Gulati V, et al. Obesity, and not obstructive sleep apnea, is responsible for increased serum hs-CRP levels in patients with sleep-disordered breathing in Delhi. Sleep Med. 2008 Jan;9(2):149-56.

12. Ciccone MM, Scicchitano P, Zito A, Cortese F, Boninfante B, Falcone VA, et al. Correlation between inflammatory markers of atherosclerosis and carotid intima-media thickness in Obstructive Sleep Apnea. Molecules. 2014 Jan;19(2):1651-62.

13. Kheirandish-Gozal L, Gozal D. Obstructive sleep apnea and inflammation: proof of concept based on two illustrative cytokines. Int J Mol Sci. 2019 Jan;20(3):459.

14. Li K, Wei P, Qin Y, Wei Y. Is C-reactive protein a marker of obstructive sleep apnea?: a meta-analysis. Medicine (Baltimore). 2017 May;96(19):e6850.

15. Iftikhar IH, Bittencourt L, Youngstedt SD, Ayas N, Cistulli P, Schwab R, et al Comparative efficacy of CPAP, MADs, exercise-training, and dietary weight loss for sleep apnea: a network meta-analysis. Sleep Med. 2017 Feb;30:7-14.

16. Mohammadieh A, Sutherland K, Cistulli PA. Sleep disordered breathing: management update. Intern Med J. 2017 Nov;47(11):1241-7.

17. Steiropoulos P, Kotsianidis I, Nena E, Tsara V, Gounari E, Hatzizisi O, et al. Long-term effect of continuous positive airway pressure therapy on inflammation markers of patients with obstructive sleep apnea syndrome. Sleep. 2009 Apr;32(4):537-43.

18. Dorkova Z, Petrasova D, Molcanyiova A, Popovnakova M, Tkacova R. Effects of continuous positive airway pressure on cardiovascular risk profile in patients with severe obstructive sleep apnea and metabolic syndrome. Chest. 2008 Oct;134(4):686-92.

19. Guo Y, Pan L, Ren D, Xie X. Impact of continuous positive airway pressure on C-reactive protein in patients with obstructive sleep apnea: a meta-analysis. Sleep Breath. 2013 May;17(2):495-503.

20. Stradling JR, Craig SE, Kohler M, Nicoll D, Ayers L, Nunn AJ, et al. Markers of inflammation: data from the MOSAIC randomised trial of CPAP for minimally symptomatic OSA: Table 1. Thorax. 2015;70(2):181-2.

21. Jullian-Desayes I, Joyeux-Faure M, Tamisier R, Launois S, Borel AL, Levy $\mathrm{P}$, et al. Impact of obstructive sleep apnea treatment by continuous positive airway pressure on cardiometabolic biomarkers: a systematic review from sham CPAP randomized controlled trials. Sleep Med Rev. 2015 Jun;21:23-38.

22. Johns MW. A new method for measuring daytime sleepiness: the epworth sleepiness scale. Sleep. 1991 Dec;14(6):540-5.

23. Wali SO, Al-Mughales J, Alhejaili F, Manzar MD, Alsallum F, Almojaddidi $\mathrm{H}$, et al. The utility of proinflammatory markers in patients with obstructive sleep apnea. Sleep Breath. 2020 Jul 23; [Epub ahead of print]. DOI: https://doi.org/10.1007/s11325-020-02149-3

24. Berry RB, Gamaldo CE, Harding SM, Lloyd RM, Marcus CL, Vaughn BV. The AASM manual for the scoring of sleep and associated events: rules, terminology and technical specifications: version 2.3. Darien, IL: American Academy of Sleep Medicine (AASM); 2017.

25. American Academy of Sleep Medicine (AASM). International classification of sleep disorders. Darien, IL: AASM; 2014.

26. Kushida CA, Chediak A, Berry RB, Brown LK, Gozal D, Iber C, et al. Clinical guidelines for the manual titration of positive airway pressure in patients with obstructive sleep apnea. J Clin Sleep Med. 2008 Apr;4(2):157-71.

27. Kim J, Lee SJ, Choi KM, Lee SK, Yoon DW, Lee SG, et al. Obstructive sleep apnea is associated with elevated high sensitivity C-reactive protein levels independent of obesity: Korean genome and epidemiology study. PLoS One. 2016 Sep;11(9):e0163017.

28. Wali SO, Abaalkhail B, AlQassas I, Alhejaili F, Spence DW, Pandi-Perumal SR. The correlation between oxygen saturation indices and the standard obstructive sleep apnea severity. Ann Thorac Med. 2020 Apr/Jun;15(2):70-5.

29. Drummond M, Winck J, Guimarães J, Santos AC, Almeida J, Marques J. Long term effect of autoadjusting positive airway pressure on C-reactive protein and interleukin- 6 in men with obstructive sleep apnoea syndrome. Arch Bronconeumol. 2009 Dec;45(12):577-84.

30. Kurt OK, Yildiz N. The importance of laboratory parameters in patients with obstructive sleep apnea syndrome. Blood Coagul Fibrinolysis. 2013 Jun;24(4):371-4.

31. Svensson M, Venge P, Janson C, Lindberg E. Relationship between sleepdisordered breathing and markers of systemic inflammation in women from the general population. J Sleep Res. 2011 Apr;21(2):147-54.

32. Lin QC, Chen LD, Yu YH, Liu KX, Gao SY. Obstructive sleep apnea syndrome is associated with metabolic syndrome and inflammation. Eur Arch Oto Rhino Laryngol. 2014 Apr;271(4):825-31.

33. Guven SF, Turkkani MH, Ciftci B, Ciftci TU, Erdogan Y. The relationship between high-sensitivity-C-reactive protein levels and the severity of obstructive sleep apnea. Sleep Breath. 2011 Mar;16(1):217-21.

34. Wessendorf T, Thilmann A, Wang YM, Schreiber A, Konietzko N, 
Teschler H. Fibrinogen levels and obstructive sleep apnea in ischemic stroke. Am J Respir Crit Care Med. 2000 Dec;162(6):2039-42.

35. Yardim-Akaydin S, Caliskan-Can E, Firat H, Ardic S, Simsek B. Influence of gender on C-reactive protein, fibrinogen, and erythrocyte sedimentation rate in obstructive sleep apnea. Antiinflamm Antiallergy Agents Med Chem. 2014 Mar;13(1):56-63.

36. Kohler M, Stradling JR. Mechanisms of vascular damage in obstructive sleep apnea. Nat Rev Cardiol. 2010 Dec;7(12):677-85.

37. Altintas N, Mutlu LC, Akkoyun DC, Aydin M, Bilir B, Yilmaz A, et al. Effect of CPAP on new endothelial dysfunction marker, endocan, in people with obstructive sleep apnea. Angiology. 2016 Apr;67(4):364-74.

38. Dediu GN, Diaconu CC, Rujinski SD, Iancu MA, Balaceanu LA, Dina I, et al. May inflammatory markers be used for monitoring the continuous positive airway pressure effect in patients with obstructive sleep apnea and arrhythmias?. Med Hypotheses. 2018 Apr;115:81-6.

39. Kumor M, Bielicki P, Przybyłowski T, Rubinsztajn R, Zieliński J, Chazan R. Three month continuous positive airway pressure (CPAP) treatmetn decreases total LDL-cholesterol levels but does not affect serum homocysteine and leptin levels in patients with obstructive sleep apnea syndrome (OSAS) without co-existent ischaemic heart disease (IHD). Pneumonol Alergol Polska. 2011 Jan;79(3):173-83.
40. Trzepizur W, Cortese R, Gozal D. Murine models of sleep apnea: functional implications of altered macrophage polarity and epigenetic modifications in adipose and vascular tissues. Metabolism. 2018 Jul;84:44-55.

41. Cortese R, Gileles-Hillel A, Khalyfa A, Almendros I, Akbarpour M, Khalyfa AA, et al. Aorta macrophage inflammatory and epigenetic changes in a murine model of obstructive sleep apnea: potential role of CD36. Sci Rep. 2017 Feb; 7:43648.

42. Mansour HAKA, Fathy A, Aref H. Effect of nasal continuous positive airway pressure on inflammatory mediators in patients with overlap syndrome. Egypt J Ear Nose Throat Allied Sci. 2011;12(2):99-104.

43. Nural S, Günay E, Halici B, Celik S, Ünlü M. Inflammatory processes and effects of continuous positive airway pressure (CPAP) in overlap syndrome. Inflammation. 2013;36(1):66-74.

44. Mermigkis C, Bouloukaki I, Mermigkis D, Kallergis E, Mavroud E, Varouchakis G, et al. CRP evolution pattern in CPAP-treated obstructive sleep apnea patients. Does gender play a role?. Sleep Breath. 2011 Sep;16(3):813-9.

45. Liu B, Taioli E. Seasonal variations of complete blood count and inflammatory biomarkers in the US population - analysis of NHANES data. PLoS One. 2015;10(11):e0142382. 